\title{
Zur interkulturellen Kommunikation in kirchlicher Sicht
}

\author{
von Franz-Josef Eilers
}

Der Fachbereich interkultureller Kommunikation ist innerhalb der Kommunikationswissenschaft relativ neu, auch wenn er der Sache nach besteht, seitdem verschiedene Kulturen auf der Erde miteinander im Austausch sind. Die Begegnungen etwa zwischen Griechen und Römern und Germanen können ebenso unter kommunikativen Gesichtspunkten gesehen werden wie ein großer Teil der Missionsgeschichte der christlichen Kirchen. Zur wachsenden Entfaltung dieses Bereiches innerhalb der Kommunikationswissenschaft haben seit Mitte der sechziger Jahre wohl drei Entwicklungen beigetragen.

Einmal beschäftigte man sich vor allem in Nordamerika um so mehr mit solchen Fragen, als man sich der verschiedenen Kulturen völkischer Minoritäten innerhalb des eigenen Landes (Farbige usw., auch französisch- und englischsprachige Teile Kanadas) bewußt wurde. Hier ist aber auch allgemein die wachsende Mobilität zwischen den Völkern und Kulturen in moderner Zeit zu nennen. Vor allem die Kollegen der Abteilungen für sprachliche Kommunikation in den USA waren es, die sich zunächst dieses Aufgabenbereiches annahmen. So hat im Dezember 1970 die amerikanische "Speach Communication Association" eine eigene "Commission for International and Intercultural Communications" begründet, die in der Folge eines Austauschprogramms $z$ wischen auswärtigen Universitäten entstand ${ }^{1}$.

$Z_{\text {weitens }}$ wird man die allgemeine Entwicklung innerhalb der Kommunikationswissenschaft vom Journalismus über die Massenmedien zur Kommunikation nennen müssen. Mehr und mehr wuchs in den letzten Jahrzehnten die Erkenntnis, daß die sogenannten Massenmedien nur ein Teil menschlicher Kommunikation sind und daß es durchaus auch eine öffentliche Aussage, Publizistik, gibt, die ohne die Massenmedien auskommen könnte, was etwa die Publizistik schriftloser Kulturen zeigt ${ }^{2}$.

Die Breite eines Kommunikationsspektrums, wie es etwa der sogenannte MacBrideReport der Unesco zeigt ${ }^{3}$, wird erst aus solcher Entwicklung verständlich.

Als Drittes aber wird man aus dem Bereich Kommunikation und Entwicklung auch auf die bittere Erfahrung hinweisen muissen, daß der simple Einsatz von Massenmedien allein in den Entwicklungsländern noch keine automatischen Erfolge bringt. Nach etlichen Jahren des Mißerfolges dämmert mehr und mehr die Erkenntnis, daß alle Kommunikation bedingt wird durch die Kultur, und nur wenn der Kommunikator sich der kulturellen Voraussetzungen des Rezipienten bewußt ist, kann es zu einem wirklichen Austausch kommen. Hier sind Überlegungen von Wilbur Schramm aus dem Jahre $1964^{4}$ und seine eigene Rückschau dreizehn Jahre später ${ }^{5}$ besonders eindrucksvoll. Der Weg von der Massenmedieneuphorie zur Bedeutung sogenannter traditioneller Kommunikation und ihrer traditionellen Kommunikationsmittel kann nicht mehr übersehen oder gar zurückgegangen werden.

\section{Interkulturelle Kommunikation}

Bei einem jungen Forschungsgebiet ist es verständlich, daß von Anfang an nicht unbedingt schon alle Definitionen klar gefaßt sind. So wird oft der Ausdruck der „interkulturellen Kommunikation" mit dem der "crosscultural communications" gleichge- 
setz $t^{\natural}$, während bei „intrakultureller Kommunikation“ schon im Wort deutlich wird, $\mathrm{da} ß$ es sich um die Kommunikation innerhalb einer Kultur handelt. Bei „internationaler Kommunikation" muß man bedenken, daß Nationen sich durchaus nicht unbedingt mit den Bereichen einer Kultur decken?. Seltener wird der Ausdruck „transkulturelle Kommunikation" gebraucht.

Interkulturelle Kommunikation kann man umschreiben als die publizistische Interaktion zwischen Mitgliedern verschiedener Kulturen ${ }^{8}$. Sie geschieht immer dann, wenn beim Austausch von Informationen und Meinungen der Kommunikator und der Rezipient verschiedenen Kulturen angehören und von diesen bestimmt sind ${ }^{9}$.

Aus diesem Begriff und ähnlichen Definitionen wird deutlich, daß interkulturelle Kommunikation mehr als andere Bereiche der Kommunikationswissenschaft interdisziplinär gesehen und ausgerichtet werden muß. Nicht nur wird man näher feststellen müssen, was unter Kultur $\mathrm{zu}$ verstehen ist ${ }^{10}$, sondern man wird sich auch der Verschiedenheit kultureller Voraussetzungen und deswegen verschiedener Folgerungen auch für die Kommunikation bewußt sein ${ }^{11}$. Ganze Wissenschaftsbereiche müssen unter kommunikativem Aspekt neu gesehen und eingebracht werden. Maletzke nennt hier als wichtigste die Wissenschaft von der Politik, die empirische Kulturanthropologie, Sozialpsychologie und Soziologie ${ }^{12}$. Für den kirchlichen Bereich sind hier aber auch die Theologie und dabei vor allem die Missiologie und die sogenannte „Dritte Welt-Theologie" zu nennen.

\section{Kommunikation des christlichen Glaubens}

Bereits vor der Entwicklung eines eigenen Fachbereiches interkultureller Kommunikation in der Kommunikationswissenschaft waren sich vor allem evangelische Missionswissenschaftler der Bedeutung der Kommunikation für die Vermittlung christlichen Glaubens zwischen verschiedenen Kulturen bewußt. Autoren wie Hendrik Kraemer und Eugene A. Nida sind dafür in unserem Jahrhundert ebenso ein Beleg wie, zumindest unter dem Aspekt der Kommunikationspraxis, alle Bemühungen bereits frühchristlicher und mittelalterlicher Homiletik und Mission. Die Begründung von Druckereien in und für die Verkündigung des dhristlichen Glaubens gehört hierher ebenso $^{13}$ wie die Ubertragung von Grundsätzen griechisch-römischer Rhetorik in die christliche Verkündigung, wie sie Augustinus bereits im vierten Buch seiner „Doctrina Christiana" vollzogen hat.

Hendrik Kraemer verfaßte 1956 seine Uberlegungen zur Kommunikation des christlichen Glaubens, die zu einem Standardwerk geworden $\operatorname{sind}^{14}$, und Nida hat sich sein ganzes Leben vor allem mit der Frage beschäftigt, wie die Botschaft der Bibel den verschiedenen Menschen kulturgerecht dargeboten werden könne. Dabei geht es ihm nicht allein um sprachwissenschaftliche Fragen der wörtlichen Übersetzung, sondern tatsächlich um die lebendige Kommunikation zwischen den Kulturen, die etwa die Struktur der Kommunikation, die Symbole und ihre Bedeutung, die Sozialstruktur, psychologische Aspekte und den gesamten kulturellen Kontext ebenso berücksichtigt wie die Dynamik kommunikativer Vorgänge in der Begegnung verschiedener Kulturen $^{15}$.

Die Schwierigkeit interkultureller Kommunikation für den christlichen Glauben wird in dem von Nida begonnenen und inzwischen von anderen weiterentwickelten sogenannten Drei-Kulturen-Modell der Kommunikation deutlich ${ }^{16}$ : Die Offenbarung des christlichen Glaubens geschah als geschichtliches Ereignis in einer Kultur, die die 
biblischen Aussagen bestimmt. Diese Offenbarung wurde von den abendländischen Kulturen aufgenommen, weiterentwickelt und soll jetzt im missionarischen Prozeß wieder anderen Kulturen weitervermittelt werden. So stehen der christliche Kommunikator, aber auch die immer noch weithin abendländisch bestimmten christlichen Kirchen vor der Frage: „How can I, who was born and brought up in one culture take truth out of the Bible which was addressed to people in a second culture, and communicate it to people who belong to a third culture without either falsifying the message or rendering it unintelligible ${ }^{\text {"17 }}$.

Das Bewußtsein dieses "drei-kulturalen“ Vorgangs ist vor allem aber in der Neuzeit und seit dem $Z_{\text {weiten }}$ Vatikanischen Konzil gewachsen. Christlicher Glaube wird nicht exportiert wie ein europäisches Produkt. Hier hat das Missionsdekret „Ad Gentes" des $Z_{\text {weiten }}$ Vatikanischen Konzils in der Betonung der Lokalkirchen ebenso neue Schwerpunkte und Maßstäbe gesetzt wie die Pastorale Konstitution über die Kirche in der Welt "Gaudium et spes" ${ }^{18}$, welche Papst Paul VI. in seinem Apostolischen Schreiben „Evangelii Nuntiandi“ vom 8. Dezember 1975 aufgegriffen hat ${ }^{19}$.

Auch das mit Recht wachsende kulturelle Selbstbewußtsein der Menschen in der sogenannten Dritten Welt stellt die Kirche hier in eine besondere Pflicht.

\section{Theologie im Kontext}

Bernard Lonergan hat als letztes Kapitel in seinem Standardwerk über „Methode in Theologie" einen eigenen Abschnitt über Kommunikation, wo er feststellt, daß alle vorhergehenden Uberlegungen seines Buches keine Frucht bringen ohne Kommunikation $^{20}$. Antonio B. Lambino greift diese Uberlegungen Lonergans in seinem Bemühen auf, sich mit der theologischen Methode der Kontextualisierung in Asien auseinanderzusetzen. In Anlehnung an Lonergan versteht er „das Verhältnis von Theologie und Tradition zur zeitgenössischen theologischen Reflexion als das Verhältnis von ,Systematik' zur ,Kommunikation" “.

Dabei hat „die Systematik die Aufgabe, ein Verständnis der großen Lehren des Christentums voranzutreiben ... Durch die Systematik wird eine genauere und vertiefte Erklärung der grundlegenden Wahrheiten des christlichen Glaubens erreicht. Die Kommunikation dagegen hat ... mit der Theologie in ihren Beziehungen zur Umwelt zu tun. An dieser Stelle macht sich die Theologie die, Tagesordnung der Welt zu eigen, die Lebensfragen des menschlichen Lebens. Diese Begegnung bringt Einsichten in die Bedeutung des Glaubenslebens inmitten der weltlichen Wirklichkeiten mit sich. Lonergan hält die Kommunikation für die Frucht des gesamten Prozesses des Theologisierens ... Die Systematik bereichert den Hintergrund für das Verstehen des Glaubens, einen Horizont, auf dem das Leben und seine Fragen angegangen werden können, Kommunikation bezieht sich auf die Dynamik dieser Interaktion zwischen dem Leben und dem Glauben ... ${ }^{\text {a21 }}$.

Hier zeigt sich in der Entwicklung einer Theologie im Kontext in den Ländern und Kontinenten der sogenannten Dritten Welt ${ }^{22}$ ein aufschlußreiches "Angewiesen sein" auch auf Uberlegungen und Erfahrungen der interkulturellen Kommunikation. Christliche Verkündigung kann nur dort "erfolgreich" geschehen, wo die kommunikativen Gegebenheiten einer Kultur ernst genommen, erkannt und in die Umsetzung systematischer Theologie in Raum und Zeit dieser Kultur hier und heute eingebracht werden. Hier geht es dann nicht mehr allein um publizistische Methoden oder Techniken im Gebrauch von Kommunikationsmitteln zur Verkündigung des christlichen Glaubens. Denn „anstatt nur die Offenbarung und die Überlieferung als Ausgangs- 
punkt zu benutzen, wie es die klassische Theologie im allgemeinen getan hat, muß sie (d. h. die "Theologie der Zeichen der Zeit" Vf.) von den Tatsachen und Fragen ausgehen, die aus der Kirche und der Geschichte abgeleitet werden ..., wenn die Kirche sich mit den wirklichen Fragen der modernen Welt auseinandersetzen und versuchen will, auf diese eine Antwort zu geben" ${ }^{23}$. Was der philippinische Theologe C. G. Arevalo hier mehr allgemein ausdrückt, meint er vor allem für die Aufgabe der Theologie und Kirche in seiner asiatischen Heimat und allgemein der sogenannten Dritten Welt. Dabei geht es um einen doppelten Schritt:

Einmal die Entdeckung der Frohbotschaft in ihrer überlieferten Form, dann aber die Umsetzung dieser Botschaft in neue kulturelle Formen, die den kommunikativen Möglichkeiten der betreffenden Kultur entsprechen. Das geht dann deswegen weit über technische Möglichkeiten der Kommunikation hinaus, weil die Kommunikation einer Kultur u. a. bestimmt ist von einer Fülle von Verhaltensweisen, von Wegen des Denkens, der Weltanschauung, von Ausdrucksmöglichkeiten der Symbolik und der Sprache und den Strukturen des gesellschaftlichen Lebens ${ }^{24}$.

Wenn Beheimatung christlichen Glaubens in den Kulturen unserer Zeit eine vordringliche Aufgabe der Weltkirche ist, dann wird man aus der Kommunikationswissenschaft dem Bereich der interkulturellen Kommunikation besondere Bedeutung beimessen müssen ${ }^{25}$.

\section{Anmerkungen}

1 Vgl. Michael H. Prosser: The Birth of a Discipline. In: Nemi C. Jain, Michael H. Prosser, Melvin H. Miller (Ed.): Intercultural Communication, Proceedings of the Speach Communications Association, Summer Conference X (mimeographed). New York 1974, S. 9 ff.

Prosser nennt hier als erstes Buch zum Themenbereich das Werk von Alfred Smith: Communication and Culture. New York 1966.

2 Vgl. Franz-Josef Eilers: Zur Publizistik sdhriftloser Kulturen in Nordost-Neuguinea. St. Augustin 1967.

s Viele Stimmen - eine Welt. Kommunikation und Gesellschaft - Heute und Morgen. Bericht der Internationalen Kommission zum Studium der Kommunikationsprobleme unter dem Vorsitz von Sean MacBride an die UNESCO. Konstanz 1981.

4 Mass Media and Development. The Role of Information in the Developing Countries. Stanford 1964.

5 Communication and Development - A revaluation. In: "Communicator", New Delhi. April 1977. S. 1-4.

- Michael Prosser unterscheidet beide, indem er mit William S. Howell "cross cultural communication" als "collective communication between cultural spokesmen of different cultural groups“ definiert, während die „Intercultural Communication “ interpersonal ist und zwischen den einzelnen Mitgliedern verschiedener Kulturen stattfindet; vgl. William S. Howell: Can Intercultural Communication be taught in a classroom? In: Midhael H. Prosser: Syllabi in Intercultural Communication 1974, Charlottesville Virginia 1975. S. 2; vgl. aud, ders. The Cultural Dialogue, An Introduction to Intercultural Communication, Boston 1978. S. 293, 299.

7 Vgl. hier u. a. Gerhard Maletzke: Internationale und interkulturelle Kommunikation. Vorschläge für Forschung und Lehre. In: „Publizistik“, Konstanz 26: 1981, 345 f.

8 Vgl. K. S. Sitaram und Roy T. Cogdell: Foundations of Intercultural Communications. Columbus 1976, S. 18. 
- Richard E. Porter und Larry A.Samovar (Communicating Interculturally) definieren: "Intercultural Communication occurs whenever a message producer is a member of one culture and a message receiver is a member of another." (In: Larry A. Samovar und Richard E. Porter: Intercultural Communication: Reader Belmont, '1976, S. 4). Gerhard Maletzke nennt ähnlich den ${ }_{p}$ process of the exchange of thaughts and meaning between people of differing cultures" interkulturelle Kommunikation. (Intercultural and International Communication). In: Heinz-Dietrich Fischer und John C. Merrill: International and Intercultural Communication, New York ${ }^{2} 1976$, S. 409.

${ }^{10}$ Vgl. hier z. B. die sehr praktische Definition von Louis L. Lutzbetak: Church and Cultures. Techny 1963, S. 60 f.

11 Vgl. hier z. B. Charles H. Kraft: Christianity in Culture. A study in Dynamic Biblical Theologizing in Cross-Cultural Perspective. Maryknoll 1979, S. 60.

12 G. Maletzke: Internationale und Interkulturelle Kommunikation. In: „Publizistik" 26: $1981,346$.

13 Vgl. z. B. Willi Henkel: Die Druckerei der Propaganda Fide. Eine Dokumentation. Paderborn 1977.

14 The Communication of the Christian Faith. London 1956; deutsche Ausgabe: Die Kommunikation des christlichen Glaubens, Zürich 1958.

15 Man vgl. hier nur etwa den Inhalt seines bereits 1960 zum ersten Mal erschienenen und immer wieder nachgedruckten Buches: Message and Mission, The Communication of the Christian Faith. New York 1960 (jetzt Pasadena ${ }^{41979) . ~ D a s ~ n e u e s t e ~ v o n ~ i h m ~ z u s a m m e n ~}$ mit William D. Reyburn veröffentlichte Werk zum gleichen Thema trägt den bezeichnenden Titel: Meaning across Cultures. Maryknoll 1981.

16 Nida: Message and Mission, a. a. O., S. 46 f.; auch bei David J. Hesselgrave: Communicating Christ cross-culturally. Grand Rapids ${ }^{21979 . ~ S . ~} 72 \mathrm{ff}$.

17 John Stott in der Einleitung des von ihm und Robert T. Coote herausgegebenen Sammelbandes: Gospel and Culture. Pasadena 1979, S. X.

18 Hier etwa Nr. $57 \mathrm{ff}$.

10 Hier u. a. bes. Nr. 20.

20 Method in Theology. London ${ }^{3} 1975$. S. 355-368.

21 Antonio B. Lambino: Zur theologischen Methode in der Kontextualisierung: Kritik an einigen asiatischen Ansätzen. In: „Zeitschrift für Missions- und Religionswissenschaft", Münster $65: 1981,8 \mathrm{f}$.

22 Zur sgn. „Dritte-Welt Theologie" vgl. Ludwig Wiedenmann: Theologie der Dritten Welt: Eine Einführung. In: Ders. (Hrsg.): Den Glauben neu verstehen. Beiträge zu einer asiatischen Theologie. Freiburg 1981, S. 7-19. Ferner: Catalino G. Arevalo: Was ist kontextuelle Theologie? ebd. 20-34.

C. G. Arevalo ebd. a. a. O., S. 33.

24 Vgl. hierzu u.a. die umfangreichen Überlegungen und die entsprechende Literatur zur Inkulturation des christlichen Glaubens. Hier u. a. Michael M. Amaladoss in seinem Beitrag zum Missionswissenschaftlichen Kongreß in Manila vom 2.-7. 12.1979. Veröffentlicht in: „Vaidika Mitram", Pattanakad (Kerala) 14: 1981, 433-447.

25 Offensichtlich ist dies - überblickt man die wenigen Publikationen zu diesem Thema mehr von einigen evangelistischen Kreisen, nicht aber den großen Kirchen erkannt. Hier wäre außer dem Buch von Hesselgrave (Communicating Christ cross-culturally, Grand Rapids 1979), auch auf die Tatsache zu verweisen, daß sich das „Language \& Intercultural Research Centre" der Brigham Young Universität (der Mormonen) besonders mit Themen dieses Sachbereichs beschäfrigt. Von dort stammt auch die bisher beste Quellenübersicht H. Ned Seelye \& V. Lynn Tyler (Ed.): Intercultural Communicator Ressources. Provo 1977. 


\section{SUMMARY}

Intercultural Communication is concerned with the communicative interaction between persons of different cultures. This is within Communications Science a newly developing area, with vast interdisciplinary consequences. For modern Theology it is especially important to be placed into the context of the different cultures in order to arrive at a new local Theology. The Gospel has to be discovered in its traditional form and then to be translated into the communicative structure of a culture, which is shaped by certain ways of thinking, the worldview, the symbols and language as well as its social structure.

\section{RESUME}

La communication interculturelle s'occupe de l'interaction communicative entre des membres de cultures différentes. C'est, à l'intérieur de la science de la communication, une branche nouvellement développée, mais en grande partie définie interdisciplinairement. Pour la théologie actuelle, elle a une signification particulière si l'on regarde cette théologie en contexte avec ses cultures. On doit reconnaître la bonne nouvelle dans sa forme transmise, mais alors la classer dans les possibilités communicatives d'une culture qui, entre autre, est définie par différents chemins de pensées, d'idéologie, de symbolique, de langage et par ses formes sociales.

\section{RESUMEN}

La comunicación intercultural se ocupa de la interacción comunicativa entre miembros de diversas sulturas. Este es un nuevo terreno desarrollado dentro de la ciencia de la communicación, pero ámpliamente interdisciplinar. Tiene especial importancia para la teología de hoy, si esta teología es considerada en contextos culturales que contribuyen a una nueva teología local. La Buena Nueva debe ser reconocida en la forma en que fué transmitida, pero al mismo tiempo hay que transplantarla las posibilidades de communicación de culturas que, entre otras cosas, están condicionadas por su propio pensamiento, visión cósmica, símbolos, idioma y formas sociales. 